\title{
An Extended Cellular Automaton Model for Train Traffic Flow on the Dedicated Passenger Lines
}

\author{
Wenbo Zhao, Yongsheng Qian, Anling Zhang, Junwei Zeng, Min Wang, and Zhidan Lv \\ School of Traffic and Transportation, Lanzhou Jiaotong University, Lanzhou 730070, China \\ Correspondence should be addressed to Yongsheng Qian; qianyongsheng@mail.lzjtu.cn
}

Received 31 December 2013; Revised 17 April 2014; Accepted 23 April 2014; Published 20 May 2014

Academic Editor: Alvaro Valencia

Copyright (C) 2014 Wenbo Zhao et al. This is an open access article distributed under the Creative Commons Attribution License, which permits unrestricted use, distribution, and reproduction in any medium, provided the original work is properly cited.

\begin{abstract}
As one of the key components for the railway transportation system, the Train Operation Diagram can be greatly influenced by many extrinsic and intrinsic factors. Therefore, the railway train flow has shown the strong nonlinear characteristics, which makes it quite difficult to take further relative studies. Fortunately, the cellular automaton model has its own advantages in solving nonlinear problems and traffic flow simulation. Considering the mixed features of multispeed running trains on the passenger dedicated lines, this paper presents a new train model under the moving block system with different types of trains running with the cellular automaton idea. By analyzing such key factors as the maintenance skylight, the proportion of the multispeed running trains, and the distance between adjacent stations and departure intervals, the corresponding running rules for the cellular automaton model are reestablished herewith. By means of this CA model, the program of train running system is designed to analyze the potential impact on railway carrying capacity by various factors; the model can also be implemented to simulate the actual train running process and to draw the train operation diagram by computers. Basically the theory can be applied to organize the train operation on the dedicated passenger lines.
\end{abstract}

\section{Introduction}

Although the Chinese railway transportation has experienced a rapid development period in recent years, the railway transport capacity still cannot efficiently meet the increasing passenger and freight transportation demands. Furthermore, limited by the current freight transportation businesses, the passenger transportation system is of a single operation scheme. In order to improve the transport capacity fundamentally and ease the contradictions in running mixed passengers and freights, the passenger dedicated lines are currently being built on a large scale in China. The passenger dedicated lines have such advantages as large capacity, high speed, and high frequency, which provide more secure, more efficient, more convenient, and more comfortable services for the increasing number of passengers. However, along with some problems emerging during the train operation process, the passenger train operation plan seems to be unreasonable and could not satisfy the passenger demands to a maximum extent up to date, although the matured model and algorithm for the existing railway have the important reference values for the compilation of the train operation diagram for a passenger dedicated line. Actually, a passenger dedicated line has its unique features, and a normal compiling theory could not necessarily be suitable for it. To optimize the train operation plan, many experts in China and abroad take a large scale of research using genetic algorithm and multiobjective programming method. However, the dynamic train operation process cannot be simulated in these algorithms $[1,2]$.

In comparison to the existing railway system, the passenger dedicated line has the following unique features: (1) it has more emphasis on passenger convenience; (2) properties and types of the trains running on passenger dedicated line are different from existing railway line; (3) the speed of trains running on passenger dedicated line is limited; (4) with strict departure time, arrival time, travel speed, and punctuality, the train operation diagram of the passenger dedicated line demonstrates more flexibility; (5) valid time zone of train operation diagram of the passenger dedicated line is smaller than the existing train operation diagram [3]. 
Checking the features listed above, it is quite necessary to use the train running simulation to analyze the train flow features on a passenger dedicated line to reveal formation mechanism of nonlinear characteristics of the railway traffic system, to predict its tendency, and to control it efficiently.

In recent years, the nonlinear characteristics in traffic flow become a hot research topic. The cellular automata (CA) are nonlinear dynamical models with its divergence in space, time, and state. With more simple algorithm and higher computational efficiency, the $\mathrm{NaSch}$ model can reproduce some actual traffic phenomena. The first CA model for the traffic flow was Number 184 Rule model proposed by Wolfram [4]. In the year of 1992, Nagel and Schreckenberg [5] proposed the well-known NaSch model as a form of the promotion. Some other scholars and experts also achieved many research results concerning a variety of traffics or road conditions on that basis [6-21].

Focused on the research field of the traffic flow, some Chinese experts have also attained several achievements of the CA model in both the theoretic research and practical application. Li et al. [22, 23], who applied the NaSch model for the purpose of an analysis of train tracking and railway traffic flow for the first time, proposed a CA model for simulating the railway traffic system. Two years later, Li et al. [24] established a CA model to analyze and explore the spacetime diagram of railway traffic flow and the trajectories of the train movement. At present, the model for simulating the railway traffic system can be roughly divided into two classes: one for the moving block system and the other for the fixed block system.

For the moving block system, more and more models for simulating the railway traffic system based on the fixed block system were proposed currently [25-30]. Zhou et al. [25] simulated the traffic phenomenon of the delay propagation in a moving-like block system. Xun et al. [26] applied CA model to simulate the train running state as well as the traffic phenomenon of the delay propagation in the rail network. Fu et al. [27] proposed a CA model to simulate the tracking operation of trains in Beijing Subway Line 2. Wang et al. [28] established some sound rules to control the running process of a train and presented a new CA model with the consideration of the mixed trains and the distance between the adjacent stations to study the moving block system.

However, the above CA models cannot be used to study the operation process of trains on the passenger dedicated line whereas some of them would not stop at any overtaking stations. In addition, the station spacing, maintenance window, and punctuality rate are not considered in their studies. According to the existing models, in view of the organization characteristics for the passenger dedicated line, we establish some rules, demarcate the related parameter, and then propose a new cellular automaton model which can efficiently simulate the phenomenon of multispeed running diagram in a moving-like block system. Then we apply this model to simulate the train operation diagram on the passenger dedicated line.

The whole paper can be organized as follows. The moving automatic block is introduced in Section 2; then in Section 3, a cellular automata model is proposed to investigate the

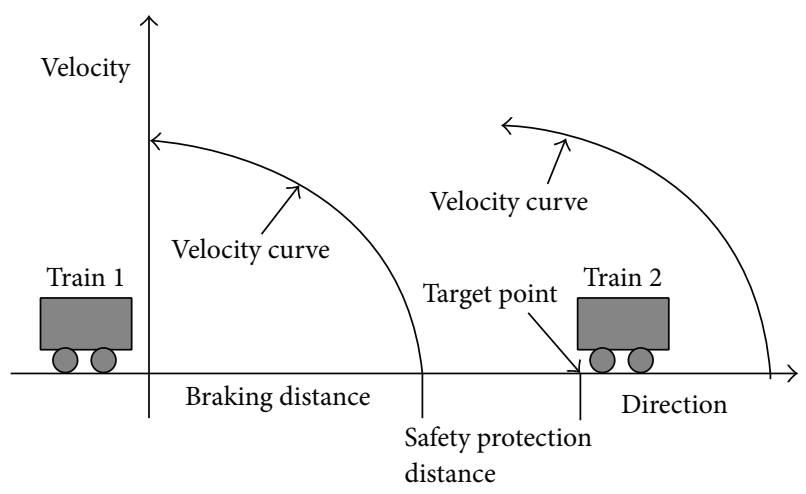

FIgURE 1: Schematic of moving block system.

mixed train tracking operation in a moving block mode, which is based on the model proposed in prior researches; in Section 4, the numerical and analytical results are presented. Finally, conclusions of this research are provided in Section 5.

\section{Moving Automatic Block}

Different from fixed autoblock system, the moving block system only takes stations and sections into consideration. In the moving block system, with no protective signals equipped at the entrance of every block, the track will not be divided into blocks. Actually, if there is enough braking distance between two adjacent trains, the train behind can increase to a higher speed to track the forward one. On this basis, not only do the trains tracking control become more flexible, but also the section capacity and efficiency can be significantly improved.

In the moving block system, the end of the preceding train, with which it keeps a certain safety distance, is the succeeding train's tracking target. According to the target distance, target speed, and train performance, the braking calculation point of the succeeding train can be calculated directly. With this method, the position of the starting point in braking process can be adjusted accordingly by the parameters of railway line and the train performance. Meanwhile, the end of the preceding train can also affect the tracking target. So the values of instantaneous space distance between the successive trains are not fixed. The moving block system is shown in Figure 1.

\section{CA Model}

The detail of cellular automata model in moving block system is built as follows.

3.1. Model Hypothesis. Based on the moving block system, there are two kinds of trains running on the railway line in a specific section, the express-trains and the lower-speed trains. Considering the same station spacing, the same length of trains, and adequate number of tracks for trains arrival and departure in one station, the lower-speed trains can stop at any overtaking station in the simulation section. In addition, 
the effect on the train operation by comprehensive maintenance window should also be considered in the model as well.

Assume the acceleration and deceleration process are not considered in the model. That is, two kinds of trains with different speeds will not enter into the system at steady speed until departure condition is satisfied. During the running time, the speed of all trains will not change in this section. But in the stations, it needs to decide whether the lowerspeed trains need to go or stop considering the express-trains tracking conditions.

3.2. Symbol and Meaning. $V_{k}$ displays the velocity of two kinds of trains, respectively, $k=1,2(k=1$ represents express-train and $k=2$ represents lower-speed train); $V_{j}(t)$ represents the real-time velocity of the train $j$ at time $t$, $V_{j}(t) \in\left\{0, V_{1}, V_{2}\right\} ; X_{j}(t)$ is the displacement of the train $j$ at time $t$; $T$ is the train interval between successive trains; $T_{z y}$ is the time requirements of basic operation including additional starting time and additional stopping time; $X_{j}(t)$ represents the position of train $j$ at time $t ; X_{j}^{k}(t)$ represents the position of train at the speed of $V_{k}$ that is closest to the initial station at time $t ; D_{j, 0}(t)$ represents the distance between train $j$ and initial station at time $t ; D_{j, 0}^{k}(t)$ represents the distance between initial station and the nearest train with $V_{k}(k=1$ represents express-train and $k=2$ represents lower-speed train); $D_{k}$ represents the distance measure for train departure $(k=1$ represents that the nearest train ahead is express-train and $k=2$ represents that the nearest train ahead is lower-speed train); $D_{j}^{k}(t)$ represents the distance between train $j$ and the contiguous train rear with $V_{k}(k=1$ represents express-train and $k=2$ represents lower-speed train); $S_{k}$ represents the reference distance standard for the lower-speed train departed from the overtaking stations $(k=$ 1 represents that the nearest train behind is express-train and $k=2$ represents that the nearest train behind is lower-speed train); $L_{\text {stop }}$ is the distance between the adjacent stations; $S_{\text {safe }}$ is the value of the minimum safety distance and $l$ is the distance of a train; $r$ is the mixture ratio (the ratio between the number of lower-speed train and the number of expresstrain).

In the model, cell neighborhood is represented as the distance of adjacent trains. Here we use distance control method to modify the running processes at each section. The quantity relationship between related parameters is

$$
\begin{array}{ll}
\text { s.t. } & D_{j, 0}(t)=X_{j}(t)-l \\
& D_{j}^{k}(t)=X_{j}(t)-X_{j-1}^{k}(t)-l \\
& D_{j, 0}^{k}(t)=X_{j}^{k}(t)-l \\
& j=1,2, \ldots, n \\
& k=1,2 .
\end{array}
$$

3.3. Train Production. In the beginning, according to a given proportion, there will generate a train randomly at the originating station with a fixed condition. If this train meets the starting condition, it starts to enter into the moving block system, with a new location updating process to be performed with a speed function $V_{j}(t)$. Otherwise, the train should wait until the starting conditions are satisfied. follows.

The judgment for the train at the initial station can be as

(1) The train interval between successive trains represented by $T$ must be larger than the basic operation time represented by $T_{z y}$ :

$$
T \geq T_{z y}
$$

(2) The outbound train will not overtake the forward train before arriving at the latest station.

The mathematical expression is as follows:

$$
\begin{array}{r}
D_{j, 0}^{1}(t)>D_{1}, \quad D_{j, 0}^{2}(t)>D_{2}, \\
D_{k}=L_{\text {stop }}-\frac{V_{1}}{V_{k}}\left(L_{\text {stop }}-S_{\text {safe }}-l\right), \\
k=1,2 .
\end{array}
$$

(3) Low-speed train leaves from overtaking station.

The mathematical expression is as follows:

$$
\begin{gathered}
D_{j}^{k}(t)>S_{k}, \quad D_{j+1}^{k}(t)>S_{\text {safe }}+l, \\
S_{k}=\left(\frac{V_{k}}{V_{2}}-1\right) L_{\text {stop }}+S_{\text {safe }}+l, \\
k=1,2 .
\end{gathered}
$$

3.4. Express-Trains Avoid Lower-Speed Trains. In order to avoid an express-train, generally speaking, the lower-speed train should stop at overtaking station. When the following conditions are satisfied, the lower-speed train can run to the next section without a stop at station for some time:

$$
\text { If } D_{j}^{2}(t)>S_{2}, \quad V_{j}(t)=V_{2}
$$

Else

$$
V_{j}(t)=0
$$

Based on this judgment, before arriving at the next overtaking station, we should continue to judge whether the lower-speed train stops at receiving-departure track or goes through the station.

\subsection{Movement. Consider the following:}

$$
X_{j}(t+1)=X_{j}(t)+V_{j}(t+1) .
$$

3.6. Operation Judgment in Condition of Comprehensive Maintenance Window. Provided that in a certain time the train operation is not allowed, the trains on the railway must stop at the adjacent station before scheduled time. 


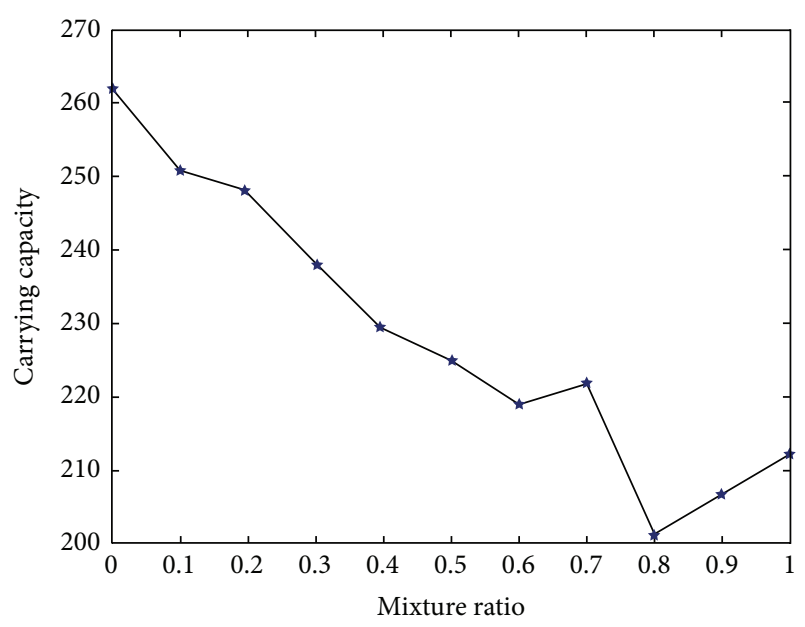

FIgURE 2: The influence on carrying capacity by mixture ratio.

\section{Simulation and Discussion}

There are four overtaking stations, one initial station, and one terminal station on the section. The speeds of an expresstrain and a lower-speed train are $198 \mathrm{~km} / \mathrm{h}$ and $80 \mathrm{~km} / \mathrm{h}$, respectively $\left(V_{1}=198 \mathrm{~km} / \mathrm{h}, V_{2}=80 \mathrm{~km} / \mathrm{h}\right)$. In order to compare simulation results with field measurements, one cellular automata iteration roughly corresponds to 1 second, and the length of a cell is $1 \mathrm{~m}$. The length of a train and safety distance are $l=500 \mathrm{~m}$ and $S_{\text {safe }}=6 \mathrm{~km}$, respectively. The basic operation time $T_{z y}=600$ cells/updating corresponds to 10 minutes actually. In addition, the maintenance window is unidirectional rectangle skylight on the alternate days with $T=7200$ cells/update corresponding to 2 hours actually. Considering open boundary condition in the model, the system will be updated by 1 second. There are 200000 time steps in one evolution stage, and here we record the time steps (24 hours) between 100000 and 186400 as one efficient date preparing for the following simulation.

4.1. The Influence on Carrying Capacity by Mixture Ratio. Figure 2 shows the value of carrying capacity in different mixture ratio. From the simulation results shown above, it can be clearly seen that as express-train train and lowerspeed train are operating on the same railway, the mixture ratio has a large influence on the carrying capacity. Only with the express-trains on the section can the values of carrying capacity be maximum. Considering mixed operation at present, it is necessary to adjust the mixture ratio properly to improve the value of carrying capacity.

\subsection{The Influence on Carrying Capacity by the Station Spacing.} Figure 3 plots same curves about the carrying capacity in different mixture ratio when taking different values of station spacing. From the simulation results shown above, it can be clearly seen that as the station spacing proceeds, the carrying capacity firstly falls suddenly and then increases rapidly. When the departure time interval is fixed, there is a close relationship between carrying capacity and station spacing.

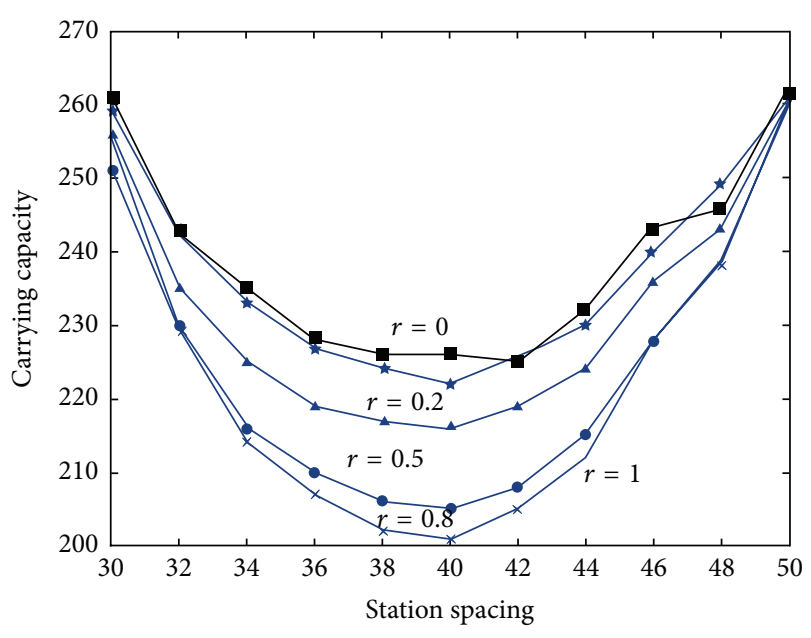

FIGURE 3: The influence on carrying capacity by station spacing in different mixture ratio ( $r$ represents the ratio of number of the express-train compared with that of the lower-speed train).

Therefore, it is necessary to adjust the values of station spacing reasonably for a higher value of carrying capacity.

The main reason behind the phenomenon could be owed to many factors. It can be clearly seen that as the $L_{\text {stop }}$ proceeds, $D_{k}$ and $S_{k}$ both increase. In the initial conditions, with large traffic density, the process of avoidance and overtaking does not much affect the total numbers of trains. Also, the velocity interference effects between different kinds of trains become obvious. In this case, affected by delay propagation at the initial station, not only should the expresstrain wait longer before departure, but also the total number of trains in the system tends to be less. After a period of running, the minimum value of carrying capacity could be seen when $L_{\text {stop }}=40 \mathrm{~km}$. When $L_{\text {stop }}$ is still on sustainable growth, lower-speed trains do not depart until avoiding most and more express-trains at the overtaking station. That is to say, when the value of $L_{\text {stop }}$ becomes larger, the exact speed difference has less effect on the running process. Most avoiding trains depart and pass through the system gradually, bringing about linear increase in carrying capacity. Therefore, it is necessary to adjust the value of station spacing reasonably for a higher value of carrying capacity.

4.3. The Influence on Carrying Capacity by the Departure Time Interval. The departure time interval is an important factor that could influence the railway carrying capacity. From the simulation results shown in Figure 4, it can be clearly seen that the value of carrying capacity decreases slowly when $T$ increases gradually. Meanwhile, with the increase of the departure time interval, the influence on carrying capacity by the proportion becomes lower and lower. In addition, with changing the value of mixture ratio, the change of carrying capacity is in accord with the trends of Figure 2, which confirms the degree of departure time interval is one of the main factors that directly affect the carrying capacity. Therefore, in order to improve the capability and efficiency of the daily production activities in a station, to bring about 


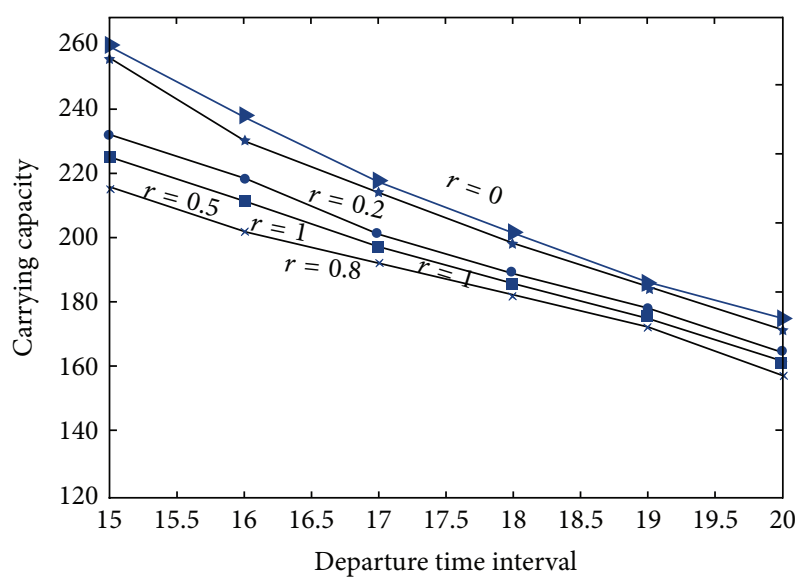

Figure 4: The influence on carrying capacity by departure time interval in different mixture ratio ( $r$ represents the ratio of number of the express-train compared with that of the lower-speed train).

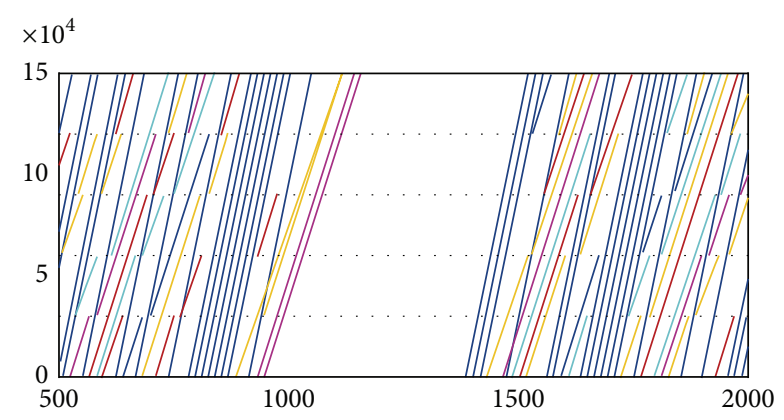

Figure 5: Train operation diagram considering maintenance window.

higher carrying capacity on the passenger dedicated line, it is important to lessen the departure time intervals.

4.4. Train Operation Diagram Considering Maintenance Window. Figure 5 shows the train operation diagram from time step $t=10000$ to time step $t=40000$ where horizontal axis and vertical axis represent the time step and the position, respectively. In the diagram, the values of carrying capacity, mixture ratio, departure time interval, and maintenance window are $30 \mathrm{~km}, 0.5,16$ minutes, and 7200 time steps, respectively. From the diagram shown above, it will be convenient to improve the maximum carrying capacity in comparison to the schemes in the train diagram establishment. Also, through appropriate selection of constraints, not only is it meaningful to optimize all the indications of train diagram and rolling stock schedule, but also it improves efficiency of rolling stock utilization plan, reducing the labor intensity for the working staff.

\section{Conclusions}

In general, this paper proposes a new CA model to simulate the railway traffic with the moving block system. The numerical simulations demonstrate that, combining with the characteristics of the moving block system, some characteristic features of the train operation can be greatly influenced by such factors as the length of train, the minimum safety distance, and so forth. We discuss thoroughly hereby the effects of station spacing, departure time interval, and the mixture ratio of different speed ratings on the railway system carrying capacity, and, in the meanwhile, we simulate the train operation diagram considering the maintenance window in the moving block system.

The simulation results are as follows.

(1) The increase of station spacing, departure time interval, and mixture ratio will result in decreased carrying capacity, but, under the influence of increasing station spacing, carrying capacity decreases firstly and then increases rapidly.

(2) In the same station spacing, there exists a big difference in the values of carrying capacity along with the different mixture ratio.

(3) The departure time interval, which basically does not subject to mixture ratio, actually has the biggest influence on carrying capacity in all the factors.

The proposed CA model is a good method to investigate the important characteristics of train operation in passenger dedicated lines. By using the simulation CA model build with MATLAB toolbox, a practical train operation diagram under the influence of the various factors can be revealed fully. However, due to some simplifying assumptions, there still exist some certain deviations between the consequences we obtained herewith and the actual railway operation system. Thus, it is worthy to study this issue deeply and further.

\section{Conflict of Interests}

The authors declare that there is no conflict of interests regarding the publication of this paper.

\section{Acknowledgments}

This work is supported by the Humanities and Social Sciences Programming Project of the Ministry of Education, China (Grant nos. 10YJA630126, 12YJC630200, and 12YJC630100) and the State Social Science Fund Project, China (Grant no. 11CJY067), and the Natural Science Foundation of Gansu Province, China (Grant nos. 1107RJYA037, 1208RJZA164, and 1308RJYA042), and The Construction of Science and Technology Key Project in Gansu Province (Grant no. JK201321) and the Young Scholars Science Foundation of Lanzhou Jiaotong University (Grant no. 2012056).

\section{References}

[1] J. Tang, Study on reasonable work-division between the passenger dedicated lines and the existing lines and optimization method [Thesis], Central South University, Changsha, China, 2008.

[2] B. Li, Simulation research on the passenger-dedicated lines transportation operation [Thesis], China Academy of Railway Sciences, Beijing, China, 2007. 
[3] M. Lv, Y. Wang, and D. Chen, "Key problems in train working diagram of passenger special line," in Proceedings of the International Conference on Logistics for Sustained Economic Development: Infrastructure, Information, Integration (ICLEM '10), pp. 3191-3197, Chengdu, China, October 2010.

[4] S. Wolfram, "Statistical mechanics of cellular automata," Reviews of Modern Physics, vol. 55, no. 3, pp. 601-644, 1983.

[5] K. Nagel and M. Schreckenberg, "A cellular automaton model for freeway traffic," Journal de Physique I, vol. 2, no. 12, pp. 22212229, 1992.

[6] K.-P. Li and Z.-Y. Gao, "Cellular automation model of traffic flow based on the car-following model," Chinese Physics Letters, vol. 21, no. 11, pp. 2120-2123, 2004.

[7] K.-P. Li, "Car deceleration considering its own velocity in cellular automata model," Communications in Theoretical Physics, vol. 45, no. 1, pp. 113-116, 2006.

[8] J.-X. Ding and H.-J. Huang, "A cellular automata model of traffic flow with consideration of the inertial driving behavior," International Journal of Modern Physics C, vol. 21, no. 4, pp. 549557, 2010.

[9] Y.-S. Qian, P.-J. Shi, Q. Zeng et al., "Analysis of the influence of occupation rate of public transit vehicles on mixing traffic flow in a two-lane system," Chinese Physics B, vol. 18, no. 9, pp. 40374041, 2009.

[10] M. Kanai, S. Isojima, K. Nishinari, and T. Tokihiro, "Ultradiscrete optimal velocity model: a cellular-automaton model for traffic flow and linear instability of high-flux traffic," Physical Review E: Statistical, Nonlinear, and Soft Matter Physics, vol. 79, no. 5, Article ID 056108, 8 pages, 2009.

[11] T. Q. Tang, H. J. Huang, S. G. Zhao, and H. Y. Shang, "A new dynamic model for heterogeneous traffic flow," Physics Letters A, vol. 373, no. 29, pp. 2461-2466, 2009.

[12] T.-Q. Tang, C.-Y. Li, H.-Y. Huang, and H.-Y. Shang, "Macro modeling and analysis of traffic flow with road width," Journal of Central South University of Technology, vol. 18, no. 5, pp. 17571764, 2011.

[13] T.-Q. Tang and W.-F. Shi, "A helicopter rescuing model in the low airspace with two telegraph poles and an electric wire," Nonlinear Dynamics, vol. 73, no. 1-2, pp. 963-970, 2013.

[14] T. Q. Tang, J. G. Li, H. J. Huang, and X. B. Yang, "A carfollowing model with real-time road conditions and numerical tests," Measurement, vol. 48, pp. 63-76, 2014.

[15] T. Q. Tang, W. F. Shi, X. B. Yang, Y. P. Wang, and G. Q. Lu, "A macro traffic flow model accounting for road capacity and reliability analysis," Physica A, vol. 392, no. 24, pp. 6300-6306, 2013.

[16] T. Q. Tang, J. He, Y. H. Wu, and L. Caccetta, "Propagating properties of traffic flow on a ring road without ramp," Physica A, vol. 396, pp. 164-172, 2014.

[17] Y.-S. Qian, W.-J. Li, J.-W. Zeng, M. Wang, J.-W. Du, and X.P. Guang, "Cellular automaton models of highway traffic flow considering lane-control and speed-control," Communications in Theoretical Physics, vol. 56, no. 4, pp. 785-790, 2011.

[18] J. P. L. Neto, M. L. Lyra, and C. R. da Silva, "Phase coexistence induced by a defensive reaction in a cellular automaton traffic flow model," Physica A, vol. 390, no. 20, pp. 3558-3565, 2011.

[19] M. Wang, Y. Qian, and X. Guang, "Improved calculation method of shortest path with cellular automata model," Kybernetes, vol. 41, no. 3-4, pp. 508-517, 2012.

[20] H.-Y. Shang and Y. Peng, "A new cellular automaton model for traffic flow considering realistic turn signal effect," Science China Technological Sciences, vol. 55, no. 6, pp. 1624-1630, 2012.
[21] J. Combinido and M. Lim, "Crowding effects in vehicular traffic," Plos ONE, vol. 7, no. 11, Article ID e48151, 2012.

[22] K. Li, Z. Gao, and B. Ning, "Cellular automaton model for railway traffic," Journal of Computational Physics, vol. 209, no. 1, pp. 179-192, 2005.

[23] B. Ning, K.-P. Li, and Z.-Y. Gao, "Modeling fixed-block railway signaling system using cellular automata model," International Journal of Modern Physics C, vol. 16, no. 11, pp. 1793-1801, 2005.

[24] K.-P. Li, Z.-Y. Gao, and L.-X. Yang, "Modeling and simulation for train control system using cellular automata," Science in China E: Technological Sciences, vol. 50, no. 6, pp. 765-773, 2007.

[25] H.-L. Zhou, Z.-Y. Gao, and K.-P. Li, "Cellular automaton model for moving-like block system and study of train's delay propagation," Acta Physica Sinica, vol. 55, no. 4, pp. 1706-1710, 2006.

[26] J. Xun, B. Ning, and K.-P. Li, "Network-based train-following model and study of train's delay propagation," Acta Physica Sinica, vol. 56, no. 9, pp. 5158-5164, 2007.

[27] Y.-P. Fu, Z.-Y. Gao, and K.-P. Li, “The characteristic analysis of the traffic flow of trains in speed-limited section for fixed-block system," Acta Physica Sinica, vol. 56, no. 9, pp. 5165-5171, 2007.

[28] M. Wang, J.-W. Zeng, Y.-S. Qian, W.-J. Li, F. Yang, and X.-X. Jia, "Properties of train traffic flow in a moving block system," Chinese Physics B, vol. 21, no. 7, Article ID 070502, 2012.

[29] Z.-C. Li, H.-J. Huang, W. H. K. Lam, and S. C. Wong, "A model for evaluation of transport policies in multimodal networks with road and parking capacity constraints," Journal of Mathematical Modelling and Algorithms, vol. 6, no. 2, pp. 239257, 2007.

[30] Z.-C. Li, W. H. K. Lam, S. C. Wong, D.-L. Zhu, and H.-J. Huang, "Modeling park-and-ride services in a multimodal transport network with elastic demand," Transportation Research Record, no. 1994, pp. 101-109, 2007. 


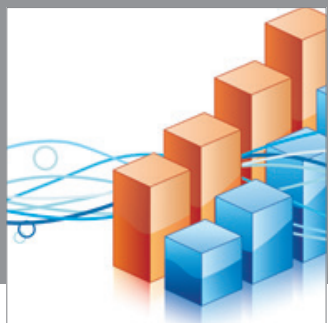

Advances in

Operations Research

mansans

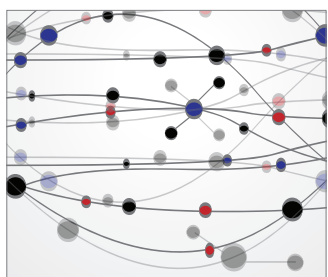

The Scientific World Journal
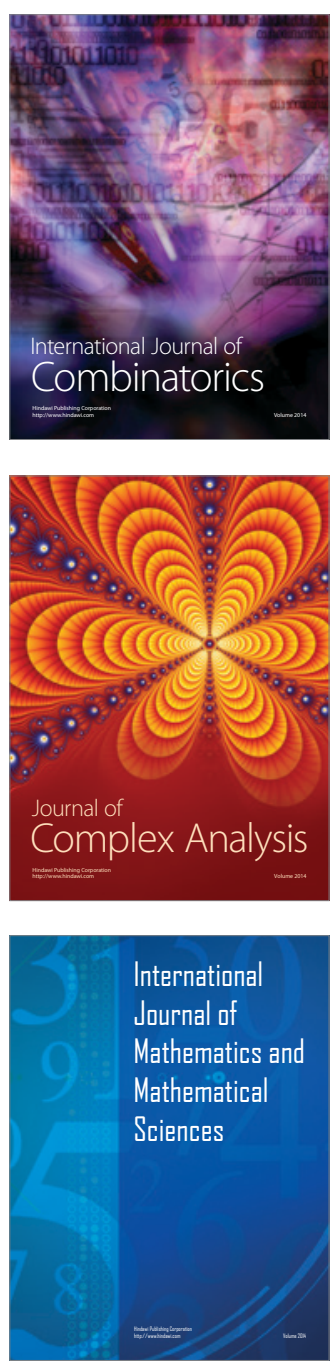
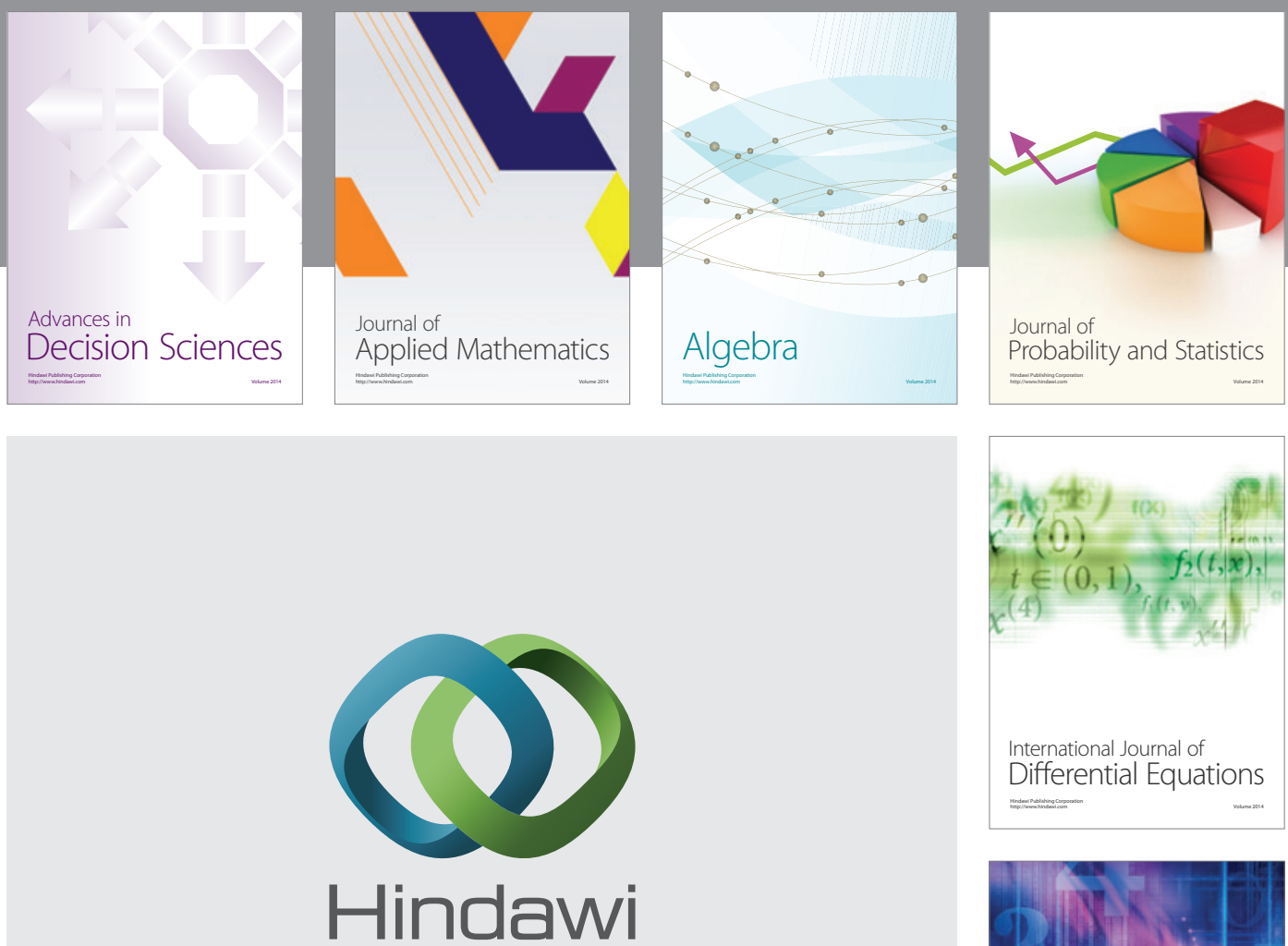

Submit your manuscripts at http://www.hindawi.com
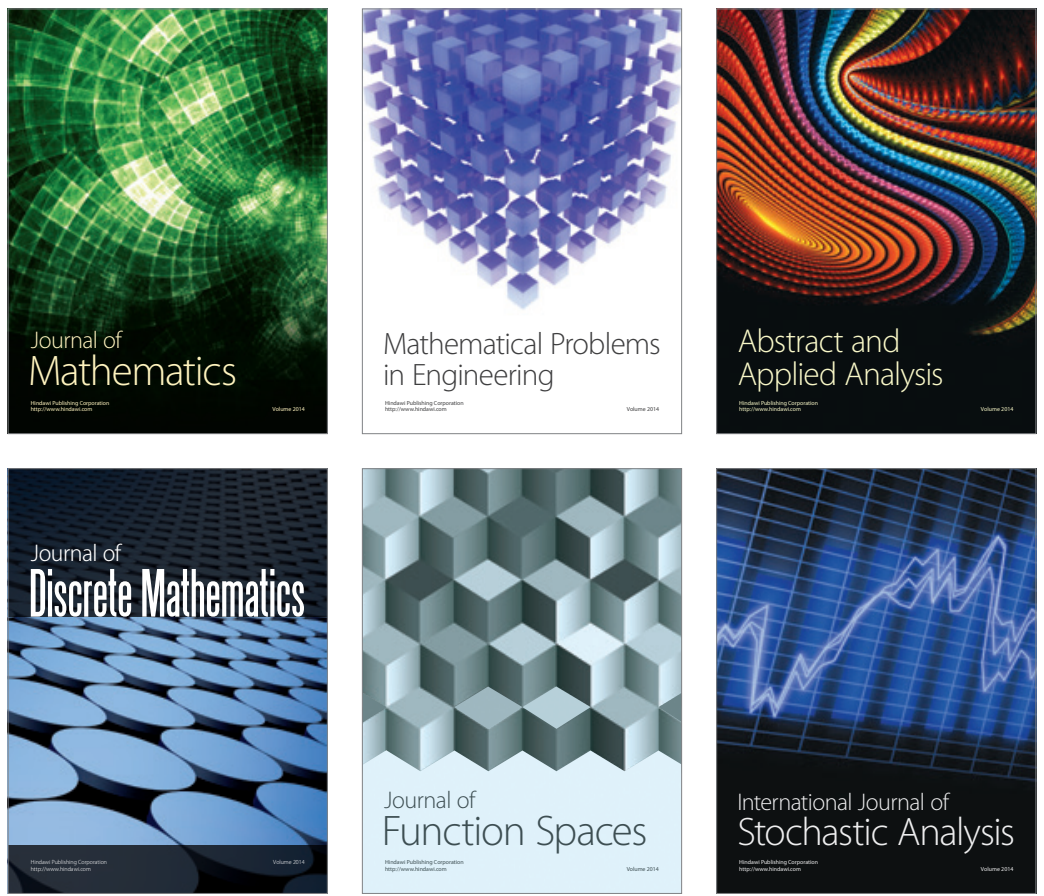

Journal of

Function Spaces

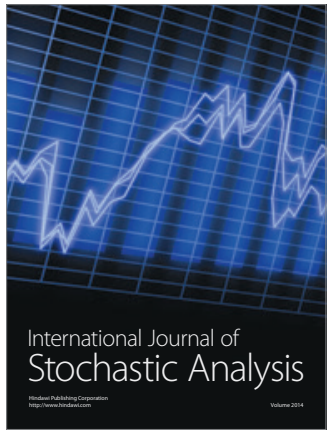

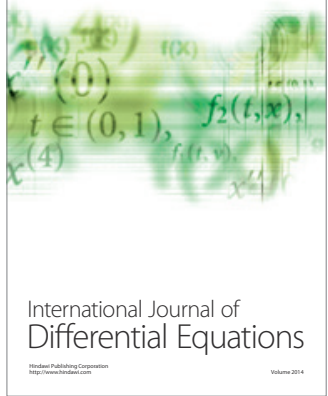
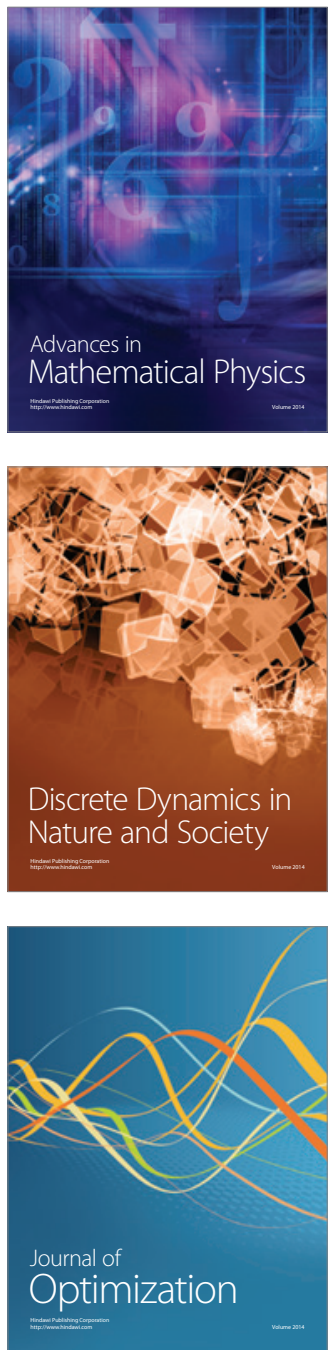